\section{The muramidase with a hole}

Enzymes concerned with the integrity of bacterial cell walls are prime targets for the design of new antibiotics. The periplasmic soluble lytic transglycosylase (SLT) from Escherichia coli pictured here (taken from Thunnissen, A-M.W.H. et al. Nature, in the press), is one such enzyme whose tertiary structure has just been determined by X-ray crystallography. This $70,000 M_{r}$ enzyme is an exomuramidase which chops up bacterial peptidoglycans into short muropeptides by hydrolysis of their glycosidic bonds.

SLT is made up of three domains of which the amino-terminal two form a striking 'super-helical' ring of $\alpha$-helices. The majority of the 'doughnut' is formed by the U-shaped $\mathrm{N}$-terminal domain (blue in the figures) which contains $22 \alpha$-helices and completed by a smaller linker-domain (green) of four helices. These domains are arranged as a two layered superhelix, each layer formed by a sheet of parallel $\alpha$-helices; the two layers being mutually anti-parallel. The sheets are forced into a curve by the insertion of an anti-parallel helix in the outer layer. A similar superhelix of helices has previously been seen in the lipovitelin-phosvitin complex from lamprey yolk (Banaszak, L., Sharrock, W. \& Timmins, P. A. Rev. Biophys. biophys. Chem. 20, 221-246 (1991)).

Surprisingly the carboxy-terminal domain (yellow) has a very similar fold to that of lysozyme despite lacking any significant sequence homology. A low resolution structure of an SLT-inhibitor complex coupled with mutagenesis studies have further emphasised the similarity by indicating that the two enzymes have analogous substrate binding sites and reaction mechanisms. The presumed localization of enzymatic activity in the lysozyme-like domain leaves open the question of the rest of the enzyme's function. It his highly likely that the $U$ - and linkerdomains increase substrate specificity by providing additional substrate-binding sites however they may be important for the mechanism of exo-enzymatic activity. By threading a proteoglycan chain through the central hole, the enzyme could remain attached after cleavage allowing it to work along the substrate, hacking off muropeptides as it goes, like some cellular logging machine.

\author{
IMAGE \\ UNAVAILABLE \\ FOR COPYRIGHT \\ REASONS
}

